providers together with components to develop self-regulation of exercise and dietary behaviour.

Discussion Revisiting complex interventions through a lens that accounts for complexity can facilitate action on a pressing public health problem like obesity and overweight. Though QCA is an inductive method, this innovative approach has enabled the identification of neglected critical aspects of WMPs, such as the nature of relationships within them, which were previously not considered to be as important as more concrete content such as dietary focus.

\section{OP84 ARE WE THERE YET? A CUMULATIVE META-ANALYSIS OF THE ACCUMULATION OF EVIDENCE FOR PHYSICAL ACTIVITY PROMOTION}

${ }^{1,2}$ R Love* ${ }^{1}$ E van Sluijs, ${ }^{1} \mathrm{~J}$ Adams, ${ }^{2} \mathrm{D}$ Humphreys. ${ }^{1}$ Centre for Diet and Activity Research (CEDAR), MRC Epidemiology Unit, University of Cambridge School of Clinical Medicine, Cambridge, UK; ${ }^{2}$ Department of Social Policy and Intervention, University of Oxford, Oxford, $U K_{i}{ }^{3}$ Department of Social Policy and Intervention, University of Oxford, Oxford, UK

\subsection{6/jech-2017-SSMAbstracts.83}

Background Despite a large evidence base and increasing number of physical activity interventions, rates of physical inactivity and associated chronic diseases are rapidly increasing globally. It is an imperative time to examine if new intervention trials have contributed novel evidence to the field. The purpose of this overview of systematic reviews is to investigate the evolution of evidence for physical activity promotion interventions over time through the analysis of changes in effect sizes for physical activity (PA) and cardiorespiratory fitness (CR) outcome measures.

Methods The search process was conducted in two stages. In the first phase, the Cochrane Central Register of Controlled Trials, MEDLINE, EMBASE, CINAHL, PsycINFO and Web of Science were searched for systematic reviews that examined the effects of interventions targeted at increasing physical activity within individuals, and included randomised controlled trials. In the second phase, primary studies included within the eligible systematic reviews were pooled. Interventions aiming to increase physical activity in healthy adults at the individual level, assessed through randomised controlled trials, were included. A cumulative meta-analysis was performed separately for interventions with PA and CR outcome measurements.

Results When assessed through cumulative meta-analysis, the totality of the evidence demonstrates that intervention effectiveness has not changed very much over the past fifteen years and that further trials are unlikely to overturn the positive and stabilised findings. The analyses indicate that the effect size for interventions became precise and stable in 2001 after the conduct of 12 physical activity (PA) and 11 cardiorespiratory (CR) outcome trials. In the time following the attainment of these thresholds of stability and precision, 70 further full scale RCTs (59 for PA and 11 for CR outcomes) have been conducted.

Conclusion Substantial evidence exists demonstrating that physical activity interventions can modify individual behaviour. However, there is limited evidence of advancements in intervention effectiveness. Given the stability and sufficiency of the evidence base, researchers are urged to shift focus towards the optimisation and scaling-up of interventions across populations.

\section{Methods}

\section{OP85 A QUALITATIVE EXPLORATION OF TRIAL-RELATED TERMINOLOGY IN A STUDY INVOLVING DEAF BRITISH SIGN LANGUAGE USERS}

${ }^{1} \mathrm{~A}$ Young ${ }^{*},{ }^{1} \mathrm{C}$ Dodds, ${ }^{1} \mathrm{R}$ Oram, ${ }^{1} \mathrm{C}$ Nassimi-Green, ${ }^{1} \mathrm{R}$ Belk, ${ }^{1} \mathrm{~K}$ Rogers, ${ }^{2} \mathrm{~L}$ Davies, ${ }^{3} \mathrm{~K}$ Lovell. 'Social Research with Deaf People, School of Health Sciences, University of Manchester and MAHSC, Manchester, UK; ${ }^{2}$ Health Services Research and Primary Care, Institute of Population Health, University of Manchester and MAHSC, Manchester, UK: ${ }^{3}$ Division of Nursing, Midwifery and Social Work, University of Manchester and MAHSC, Manchester, UK

\subsection{6/jech-2017-SSMAbstracts.84}

Background British Sign Language (BSL) is a fully grammatical, living language, independent of spoken English. Deaf BSL users are routinely excluded from clinical trials either on grounds of language and/or assumptions of confounding factors associated with disability. Few clinical trials specifically targeted at Deaf people exist internationally. Appropriate terminology in BSL for key concepts in clinical trials is needed to support informed consent.. Barriers to conceptual (mis) understanding of trial participation and sources relevant to the Deaf community are unexplored. This study set out to:

(i) Explore, in BSL, the meaning and understanding of key concepts and common vocabulary used in recruitment and informed consent to a clinical trial

(ii) Enable signs/signed expressions to emerge that are semantically accurate and support Deaf people's informed consent in any future trial

Methods A community participatory approach underpinned data collection. This comprised Deaf-led, qualitative, exploratory group discussions (19 self-selected participants in five groups), facilitated in BSL. Discussion was structured to explore the meaning of key terminology (such as trial, randomisation, consent) and through that exploration generate signed explanations of concepts to use in future trials. Data were video-recorded and analysed in BSL, using a phenomenological approach, built from the application of 4 themes and 8 sub-themes using NVIVO8 for visual data.

Results All participants were over 30 years old, with a selfdeclared 'strong Deaf identity'; BSL was their first or preferred language. Six necessary conditions for developing optimal trial information were identified. (i) developing appropriate expressions and terminology from a community basis, rather than testing out previously derived translations from a different language; (ii) paying attention to languagespecific features which support best means of expression (in the case of BSL expectations of specificity, verb directionality, handshape); (iii) accounting for bilingual influences on comprehension; (iv) deliberate orientation of information to avoid misunderstanding not just to promote accessibility; (v) sensitivity to barriers to discussion about intelligibility of information that are cultural and social in origin, rather than linguistic; (vi) the importance of using contemporary language-in-use, rather than jargon-free or plain language, to support understanding.

Conclusion These conditions need to be met to develop signed participant information for Deaf people that is: acceptable, accessible, transmitted accurately and understood as intended. They are required to address the cultural preferences and lower background knowledge of Deaf people (using signed languages) as well as in spoken/written languages when 